\title{
Switching in Adults with Aphasia
}

Switching is critical for cognitive flexibility as one adaptably shifts attention, ideas, sets and responses (Rende, 2000). Switching requires regulation of processes of current and previous tasks (reconfiguration control) with the ability to resist interference from previous tasks (interference control) (Rogers \& Monsell, 1995). Few studies have investigated switching in adults with aphasia, even though clinicians have observed their difficulty switching attention from one task to another. This may interfere with their ability to use what they learn in therapy (Kraat, 1990).

Underlying cognitive deficits were suggested to explain impaired shifts. Adults with nonfluent aphasia were trained to use three modalities (verbal, gesture, and communication board) and continued to encounter difficulty spontaneously switching between modes, especially from the verbal mode to others (Purdy et al., 1994). HelmEstabrooks (2002) found that adults with mild aphasia had difficulty switching between circles and triangles. Chiou and Kennedy (2006) examined relationships across performance in linguistic (e.g., Western Aphasia Battery, Discourse Comprehension Test) and non-linguistic tasks (e.g., WCST, Design Fluency), and a metacomprehension task that measured participants' ability to judge the accuracy of their answers. Adults with aphasia who were better judges of their answers to yes/no questions had fewer set loss errors, fewer perseverative responses and were more accurate at set switching than adults who were poor judges of their answers. Thus, maintaining and shifting sets appear to be shared processes with making confidence judgments about ones comprehension, all of which are a part of the supervisory system.

Assessment options are limited for individuals with aphasia because of the language load and involvement of multiple cognitive processes in many complex cognitive tasks (Keil \& Kaszniak, 2002). Go/no-go (GNG) tasks avoid linguistic and cognitive demands by requiring simple responses, enabling us to pinpoint switching processes. The research questions were:

1. Are adults with aphasia as accurate and as fast as healthy controls when switching between rules?

2. Are there relationships between GNG switching measures and other linguistic and nonlinguistic measures that involve switching?

\section{Participants}

\section{METHODS}

Fourteen adults with mild or moderate aphasia and fourteen age- and educationmatched healthy controls were included in this study (Table 1). All participants were native English speakers without history of neurological disease, substance abuse, and psychiatric disorder. All participants had adequate hearing, vision and attention determined from a battery of tests. Participants had to remember sequences of three letters presented visually on a note card after a $5 \mathrm{~s}$ delay to ensure their ability to hold visual information. Adults with aphasia had chronic language disability from a left hemisphere stroke (Table 2). 


\section{Stimuli and Procedures}

Two types of modified GNG tasks were designed to assess switching between rule sets in a short period of time: GNG tasks with rule-switching and GNG tasks without rule-switching. In the GNG task, a response selection process was required between either executing or inhibiting a response, triggered by a "go" stimulus or a "no-go" stimulus. A visual-auditory detection task served as a baseline measure to confirm participants' ability to detect stimuli. Predictability was an independent variable in the GNG tasks and was not the interest of this proposal: Predictable (presentation modality of test stimuli was alternating between visual and auditory) and unpredictable (modality was presented in random).

Two types of stimuli were presented on the GNG tasks: Orthographic letter stimuli and auditory stimuli (i.e., letter names) presented one at a time. Distracters which share similar visual contours or phonemes with the target stimulus were excluded.

The GNG tasks without switching required participants to follow a rule, for example, "Do not respond to O". The GNG tasks with rule-switching contained five rule shifts. A rule was specified on the computer screen (e.g., "Do not respond when you see E”) and a card with printed rule was remained in view, i.e., participants had to ignore a letter in a specified modality (visual or auditory). Rules of the task changed after every 16 stimuli and the 16 stimuli included 4 no-go stimuli (target) and 12 go stimuli (distracters). The 12 go stimuli contained four types of distracters: four novel stimuli, four target stimuli in the other modality, two last target stimuli, and two last target stimuli in the other modality. A range of interstimulus intervals (2000 ms +/- $1000 \mathrm{~ms}$ ) were used to avoid establishment of a response pattern. Stimuli were presented for 1000 (ms).

GNG tasks were created and presented with "eevoke“, a program on a laptop with a 15 inch color screen. Participants responded to a "go" stimulus by pressing a button on a game pad with their left index finger. Two speakers were placed besides the laptop.

Several neurocognitive tests were selected to assess cognitive flexibility and switching skills. Scaled scores of these tasks were obtained to explore relationships with the experimental tasks.

\section{Switching}

\section{RESULTS}

Mean switch costs, the difference in reaction times between switching and no switching, served as the dependent variable (DV) in a repeated measures group x predictability ANOVA. Adults with aphasia were slower in self-initiated control when switching from one rule to the other compared to healthy control participants collapsed across predictability $[\mathrm{F}(1,26)=6.110, \mathrm{p}=0.020]$ (Table 3).

A group x predictability x error type (new stimuli, last target) x similarity (same, different modalities) ANCOVA was generated with the number of new and last target stimuli errors (omission errors) as the DVs and numbers of omission errors in the detection task as the covariate. Adults with aphasia made more omission errors than healthy controls $[F(1,25)=20.652, p=.0001]$. One of the interaction effects (Table 3 ) revealed that adults with aphasia were also negatively affected by previous rule-sets (missed responses to last target stimuli with the same modality as the current no-go target stimuli) $[\mathrm{F}(1,25)$ $=8.454, \mathrm{p}=.008$ ]. This is evidence of perseveration in aphasia (Figure 1). 
Number of target stimuli errors (commission errors) served as the DV in a repeated measures group x predictability x similarity ANOVA. Adults with aphasia made more mistakes than healthy controls collapsed across similarity and predictability when switching rules $[\mathrm{F}(1,26)=30.528, \mathrm{p}=.00001]$ (Table 3 ). The results indicated that adults with aphasia having difficulty formulating and constructing a new rule set (reconfiguration control).

\section{Relationships Between Switching Measures}

Positive relationships among GNG rule-switching (percent correct) and several nonlinguistic switching measures (scaled scores) were found in Table 4. Linguistic and nonlinguistic cognitive flexibility (Sorting test) was not related to other switching measures. Severity of aphasia was related with some nonlinguistic switching measures (Table 5).

\section{DISCUSSION}

The findings provide evidence that adults with aphasia have difficulty switching when tested using a simple task that has little linguistic load. Predictable and unpredictable stimuli presentations influenced participants' performance to some extent.

- Adults with mild or moderate aphasia demonstrated reduced ability to flexibly switch between rules compared to healthy controls, with evidence of reduced reconfiguration and interference control.

- Rule switching may share common cognitive processes with nonlinguistic switching.

- The relevance of these findings for everyday communication will be discussed. 


\section{References}

Brookshire, R.H., \& Nicholas, L.E. (1993). The Discourse Comprehension Test. Minneapolis, MN: BRK Publishers.

Chiou H. \& Kennedy, M.R.T. (2006, February). Executive functions and Aphasia: Relationships Between Metacomprehension and Switching Behavior. $34^{\text {th }}$ Annual International Neuropsychological Society Association, Boston, MA.

Delis, D.C., Kaplan, E., \& Kramer, J.H. (2001). Delis-Kaplan Executive Function System. San Antonio, Texas: The Psychological Corporation.

Heaton, R.K., Thompson, J.A., \& Gomez, E. (1999). Wisconsin Card Sorting Test: Computer version 3 for windows research edition. Odessa, Florida: Psychological Assessment Resources, Inc.

Helm-Estabrooks, N. (2002). Cognition and aphasia: A discussion and a study. Journal of Communication Disorders, 35, 171-186.

Keil, K. \& Kaszniak, A.W. (2002). Examining executive function in individuals with brain injury: A review. Aphasiology, 16, 305-335.

Kertesz, A. (1982). Western Aphasia Battery. New York: The Psychological Corporation.

Kraat, A.W. (1990). Augmentative and alternative communication: Does it have a future in aphasia rehabilitation? Aphasiology, 4, 321-338.

Purdy, M. H., Duffy, R. J., \& Coelho, C. A. (1994). An Investigation of the communicative use of trained symbols following multimodality training. In P. Lemme (Ed.), Clinical Aphasiology: Vol.22 (pp.345-356). Austin, TX: ProEd.

Rende, B. (2000). Cognitive flexibility: Theory, assessment, and treatment. Seminars in Speech and Language, 21, 121-133.

Ridgeway, V., Robertson, L.H., \& Ward, T. (1994). Test of Everyday Attention (TEA). Suffolk, England: Thames Valley Test Company.

Rogers, R.D, \& Monsell, S. (1995). Costs of a predictable switch between simple cognitive tasks. Journal of Experimental Psychology: General, 124, 207-231. 
Table 1. Participants' demographic information

\begin{tabular}{lcc}
\hline & $\begin{array}{c}\text { Aphasia } \\
\text { mean } \pm \text { SD }\end{array}$ & $\begin{array}{c}\text { Control } \\
\text { mean } \pm \text { SD }\end{array}$ \\
\hline Age At Testing (yrs) & $63.85 \pm 11.83$ & $66.00 \pm 12.29$ \\
Gender: Male/female & $6 / 8$ & $7 / 7$ \\
Years of Education & $15.07 \pm 2.46$ & $16.21 \pm 2.05$ \\
Western Aphasia Battery & & \\
\hline $\begin{array}{l}\text { Aphasia quotient* } \\
\text { Cortical quotient* }\end{array}$ & $76.33 \pm 11.48$ & $99.70 \pm 0.23$ \\
Communicative Abilities of Daily & $77.97 \pm 8.96$ & $98.01 \pm 2.15$ \\
\hline Living* & $90.71 \pm 8.30$ & $99.00 \pm 0.00$ \\
ASHA FACS & & \\
$\quad \begin{array}{l}\text { Social communication * } \\
\text { Communication of basic needs }\end{array}$ & $5.78 \pm 1.07$ & $6.84 \pm 1.07$ \\
$\quad$ Reading, writing, number concept* & $6.57 \pm 0.66$ & $6.93 \pm 0.18$ \\
$\begin{array}{l}\text { Daily planning* } \\
\text { Overall communication } \\
\text { independence* (\%) }\end{array}$ & $5.85 \pm 0.88$ & $6.94 \pm 0.19$ \\
$\begin{array}{l}\text { Visual memory (\% correct) } \\
\text { Three letter sequences }\end{array}$ & $5.91 \pm 0.98$ & $6.98 \pm 0.06$ \\
$\quad$ Three to six letter sequences* & $86.33 \pm 10.34$ & $98.78 \pm 2.37$ \\
\hline
\end{tabular}

${ }^{*} p<.05$ 
Table 2. Descriptive Aphasia and Stroke Information.

\begin{tabular}{|c|c|c|c|c|c|}
\hline Ss & $\mathrm{AQ} / \mathrm{CQ}^{*}$ & Type & Severity & $\begin{array}{l}\text { Years post- } \\
\text { injury }\end{array}$ & Neurological Findings \\
\hline 1 & $74.40 / 82.60$ & Anomic & moderate & 1.17 & $\begin{array}{l}\text { L parietal and } \\
\text { temporal lobes infarct }\end{array}$ \\
\hline 2 & $74.40 / 75.90$ & Anomic & moderate & 5.08 & L CVA \\
\hline 3 & $74.00 / 73.90$ & Conduction & mild & 1.42 & $\begin{array}{l}\text { L posterior MCA } \\
\text { occlusion }\end{array}$ \\
\hline 4 & $67.70 / 75.10$ & Broca's & mid & 4.25 & $\begin{array}{l}\text { L insular and temporal } \\
\text { lobe MCA infarct }\end{array}$ \\
\hline 5 & $90.90 / 84.25$ & Anomic & mild & 4.00 & $\mathrm{~L}$ AVM \\
\hline 6 & $88.10 / 88.30$ & Anomic & mild & 2.50 & $\begin{array}{l}\text { L temporal } \\
\text { hemorrhage }\end{array}$ \\
\hline 7 & $69.10 / 69.50$ & Broca's & mild & 1.58 & L MCA infarct \\
\hline 8 & $61.00 / 67.35$ & Broca's & mild & 0.67 & L MCA infarct \\
\hline 9 & $93.20 / 93.20$ & Anomic & mild & 2.00 & L parietal CVA \\
\hline 10 & $93.00 / 85.80$ & Anomic & mild & 2.00 & $\mathrm{~L}$ posterior CVA \\
\hline 11 & $78.60 / 80.05$ & Anomic & moderate & 1.67 & L CVA \\
\hline 12 & 69.10/69.50 & Broca's & mild & 2.67 & L CVA \\
\hline 13 & $57.20 / 62$ & Broca's & mild & 3.33 & $\begin{array}{l}\text { L temporal-parietal } \\
\text { MCA infarct }\end{array}$ \\
\hline 14 & $77.88 / 84.10$ & Anomic & moderate & 10.92 & L CVA \\
\hline M & 76.33/77.97 & & & 3.09 & \\
\hline SD & $11.48 / 8.96$ & & & 2.59 & \\
\hline
\end{tabular}


Table 3.

Repeated Measures Analyses of Variance for the Go/no-go Tasks with Rule-switching.

\begin{tabular}{clllll}
\hline & Huynh- & & & \\
Analysis and Source & Feldt $d f$ & $F$ & $p$ & $\hat{\eta}$ \\
\hline
\end{tabular}

$\underline{\text { Switching Cost }}$

Group x Predictability ANOVA

Between subjects

$\begin{array}{lcccc}\text { Group } & 1 & 6.110 & 0.020 & 0.190 \\ \text { Error } & 26 & & & \end{array}$

Within subjects

Predictability

$1 \quad 0.034 \quad 0.855$

Group x Predictability

$1 \quad 0.204 \quad 0.655$

Error

26

New and Last Target Errors (Omission

Errors)

Group x Predictability x Error Type x

Similarity ANCOVA

Between subjects

$\begin{array}{lcccc}\text { Group } & 1 & 20.652 & 0.0001 & 0.452 \\ \text { Error } & 25 & & & \\ \text { Within subjects } & & & & \\ \text { Predictability } & 1 & 0.431 & 0.517 & \\ \text { Group x Predictability } & 1 & 1.540 & 0.226 & \\ \text { Error } & 25 & & & \\ \text { Error type } & 1 & 2.747 & 0.110 & \\ \text { Group x Error Type } & 1 & 7.017 & 0.014 & 0.219\end{array}$


Error

Similarity

Group x Similarity

Error

Predictability x Error Type

Group x Predictability x Error type

Error

Predictability x Similarity

Group x Predictability x Similarity

Error

Error Type x Similarity

Group x Error Type x Similarity

Error

Predictability x Error Type x Similarity Group x Predictability x Error Type x Similarity

Error

Target Errors (Commission Errors)

Group x Predictability x Similarity ANOVA
25

$\begin{array}{ccc}1 & 0.050 & 0.825 \\ 1 & 0.413 & 0.526 \\ 25 & & \\ & & \end{array}$

$1 \quad 0.402 \quad 0.532$

$\begin{array}{llll}1 & 5.659 & 0.025 & 0.185\end{array}$

25

$\begin{array}{lll}1 & 0.271 & 0.607\end{array}$

$\begin{array}{lll}1 & 0.139 & 0.712\end{array}$

25

$\begin{array}{llll}1 & 5.334 & 0.029 & 0.176\end{array}$

$\begin{array}{llll}1 & 8.454 & 0.008 & 0.253\end{array}$

25

$1 \quad 1.007 \quad 0.325$

$1 \quad 1.026 \quad 0.321$

26

Between subjects

Group

Error

Within subjects

Predictability

Group x Predictability

Error
1

26

$\begin{array}{ccc}1 & 0.634 & 0.433 \\ 1 & 0.000 & 1.000 \\ 26 & & \end{array}$




$\begin{array}{lccc}\text { Similarity } & 1 & 0.646 & 0.429 \\ \text { Group x Similarity } & 1 & 3.591 & 0.069 \\ \text { Error } & 26 & & \\ \text { Predictability x Similarity } & 1 & 0.461 & 0.503 \\ \text { Group x Predictability x Similarity } & 1 & 0.628 & 0.435 \\ \text { Error } & 26 & & \\ \end{array}$


Table 4.

Pearson Correlations among Accuracy Measures of the Go/no-go Tasks and Switching in Neurocognitive Tests for the Aphasia Group $(n=14)$.

\begin{tabular}{ccc}
\hline & \multicolumn{2}{c}{$\begin{array}{c}\text { Go/no-go Tasks } \\
\text { With Rule-Switching }\end{array}$} \\
\cline { 2 - 3 } & $\begin{array}{c}\text { Predictable } \\
\text { Percent Correct }\end{array}$ & $\begin{array}{c}\text { Unpredictable } \\
\text { Percent Correct }\end{array}$ \\
\hline
\end{tabular}

Go/no-go Tasks Without Rule-Switching

Predictable percent correct

0.347

0.262

Unpredictable percent correct

$-0.119$

0.425

$\underline{\text { Sorting Subtest }}$

Confirmed/unconfirmed target sorts

0.368

0.175

$\underline{\text { Trail Making Subtest }}$

Number-letter switching

$0.610 * * *$

$0.826 * * * * *$

$\underline{\text { Design Fluency Subtest }}$

Switching

0.523

$0.560 * *$

Total attempted designs

$0.672 * * * *$

0.401

Percent design accuracy

0.101

0.110

$\underline{\text { TEA Visual Elevator Subtest }}$

Raw accuracy scores

0.501

0.520

Attentional switching timing

0.556

$0.585^{*}$

Note: Correlation $<.05$ indicates statistical significance. ${ }^{*} p=.046,{ }^{* *} p$

$=.037, * * * p=.021, * * * * p=.008, * * * * * p=.00027$ 
Table 5.

Pearson Correlations among Aphasia Quotients and Cortical Quotients and Switching and No Rule-switching Measures for the Aphasia Group ( $n=14)$.

$\mathrm{AQ} \quad \mathrm{CQ}$

$\underline{\text { Western Aphasia Battery }}$

Aphasia quotient (AQ)

1

Cortical quotient (CQ)

$0.924 * * * *$

1

Go/no-go Tasks with Rule-switching

Predictable switch cost

$-0.102$

$-0.080$

Unpredictable switch cost

$-0.119$

$-0.157$

Predictable percent correct

0.406

0.435

Unpredictable percent correct

0.136

0.282

$\underline{\text { Sorting Subtest }}$

Confirmed correct sort

0.009

$-0.020$

$\underline{\text { Trail Making Subtest }}$

Number-letter switching

0.236

0.446

$\underline{\text { Design Fluency Subtest }}$

Switching

0.481

$0.628 * * *$

Total attempted designs

0.047

0.140

Percent design accuracy

0.437

0.368

$\underline{\text { TEA Visual Elevator Subtest }}$

Raw accuracy scores

$0.621^{*}$

$0.653^{* *}$

Attentional switching timing

0.411

0.528

Note: Correlation $<.05$ indicates statistical significance. ${ }^{*} p=.031,{ }^{* *} p=.021,{ }^{* * *} p$

$=.016, * * * * p=.00024$ 
Figure 1. Switching cost and detection across participants

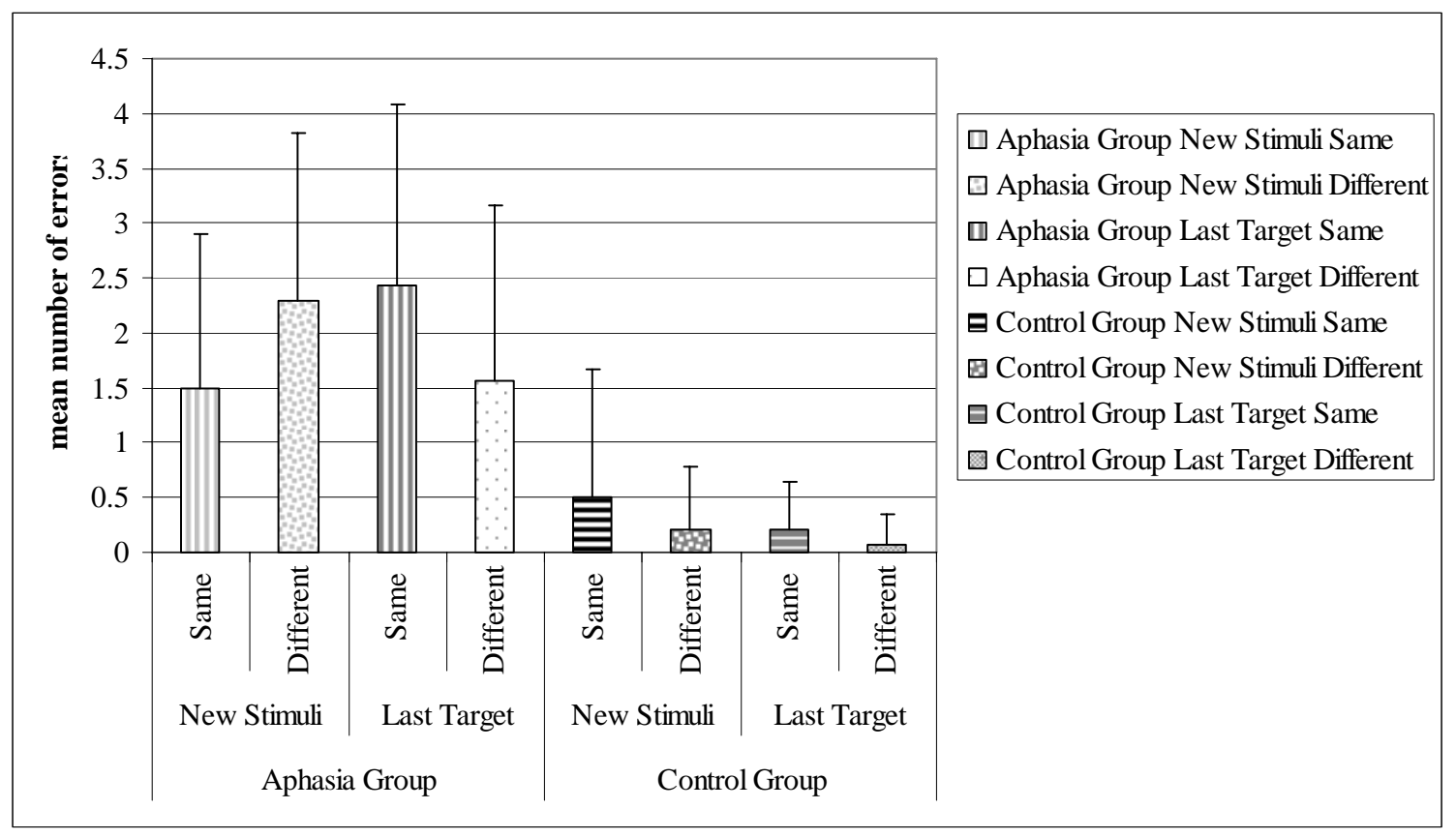

